\title{
Prognostic Value of FDG-PET/CT Parameters in Patients with Locally Advanced Rectal Cancer Treated with Neoadjuvant Approach
}

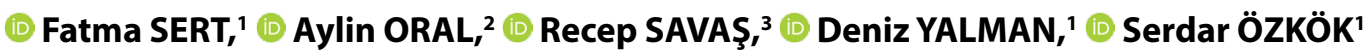 \\ 'Department of Radiation Oncology, Ege University, Faculty of Medicine, İmir-Turkey \\ ${ }^{2}$ Department of Nuclear Medicine, Ege University, Faculty of Medicine, İzmir-Turkey \\ ${ }^{3}$ Department of Radiology, Ege University, Faculty of Medicine, İzmir-Turkey
}

\begin{abstract}
OBJECTIVE
To determine the prognostic and/or predictive role of 18F-FDG PET/CT parameters, such as SUVmax, SUVmean, Metabolic Tumor Volume (MTV) and Total Lesion Glycolysis (TLG=MTVxSUVmean), for the patients with locally advanced rectal cancer (LARC) treated with neoadjuvant radiotherapy \pm chemotherapy.

\section{METHODS}

Between January 2005 and December 2016, a total of 106 patients with clinical T3-4 and/or N+ rectal cancer without distant metastasis were included in this study. Correlation between metabolic and volumetric parameters and tumor characteristics was evaluated. Prognostic factors for overall survival (OS), local recurrence-free survival (LRFS), and distant metastasis-free survival (DMFS) were analyzed.
\end{abstract}

\section{RESULTS}

The median follow-up duration for all patients was 39.0 months (range, 6-103 months). Pathologic complete response (pCR) was defined as the absence of viable cancer cells in the resected specimen (ypT0N0). pCR was achieved in $17 \%$ of all cases (18/106). There was a weak correlation between SUVmax of primary tumor and MTV $([\mathrm{r}]=0.238 ; \mathrm{p}<0.001)$. However, SUVmax of primary tumor and TLG were significantly correlated $(\mathrm{r}=0.538 ; \mathrm{p}<0.001)$. Neither SUVmax nor SUVmean was affected by patient and tumor characteristics. Posttreatment extensive stage of disease $(\mathrm{p}=0.013)$, absence of concomitant CT ( $\mathrm{p}=0.012)$, MTV $\geq 14.65 \mathrm{~cm}^{3}(\mathrm{p}=0.008)$, and TLG $\geq 117.00$ ( $\mathrm{p}=0.023$ ) were unfavorable prognostic factors for OS on multivariate analysis.

\section{CONCLUSION}

Although FDG-PET is not a standard imaging modality for the treatment of rectal cancers, a negative effect of high MTV and TLG on OS was shown in our study. We should consider more intense treatment approaches for tumors with high MTV and TLG values.

Keywords: FDG-PET/CT; locally advanced rectal cancer; prognostic factors; radiochemotherapy. Copyright $\odot$ 2020, Turkish Society for Radiation Oncology 


\section{Introduction}

For locally advanced rectal cancer (LARC) patients, neoadjuvant radiochemotherapy (nRCT) followed by total mesorectal excision (TME) is the standard treatment.[1,2] Studies have shown that nRCT provides better local control with lower toxicity rates than adjuvant treatment approaches.[3,4] On the other hand, the patients show heterogeneous responses to treatment concerning pathological response and survival. Approximately $10-30 \%$ of the patients show pathologic complete response, $40-45 \%$ show variant tumor regression, and the remaining $20-30 \%$ have no response to nRCT.[5-7] In addition, the 10-year cumulative incidence of local relapse was $7.1 \%$ in the preoperative arm of the German CAO/ARO/AIO-04 randomized phase 3 trial.[4] Therefore, additional efforts are required to predict the prognostic factors for these high-risk patients.

Several imaging modalities are currently used for staging the disease and monitoring the response to nRCT in patients with LARC. Concomitant use of conventional computed tomography (CT), magnetic resonance imaging (MRI) has been shown to be effective for local staging of rectal cancer by accurately determining the $\mathrm{T} 4$ stage with circumferential resection margin involvement. [8] These morphological evaluations have no relation with either prognosis or treatment response. Positron emission tomography (PET) using 18 fluorodeoxyglucose (18F-FDG) has been reported to be valuable functional imaging modality that has demonstrated distinguished capabilities in fields of primary cancer detection, planning and monitoring treatment, prognosis prediction, early detection of recurrent disease, and the diagnosis of regional lymph node and distant metastasis in various cancers.[9-11] Several parameters of 18F-FDG PET/ CT, such as the SUVmax, SUVmean, SUVpeak, tumor functional longitudinal length, metabolic tumor volume (MTV), and total lesion glycolysis (TLG) have been suggested to be useful prognostic indicators in cancer patients.[12,13] Among these parameters, volumetric parameters, such as MTV and TLG, are expected to help in measuring volumetric tumor burden, and they could have roles as prognostic factors in malignant disease.[14]

Is the present study aims to determine the prognostic and predictive role of $18 \mathrm{~F}-\mathrm{FDG}$ PET/CT parameters, such as SUVmax, SUVmean, MTV and TLG, for the patients with LARC treated with nRCT.

\section{Materials and Methods}

\section{Eligibility Criteria}

Between January 2005 and December 2016, a total of 106 patients with clinical T3-4 and/or N+ rectal cancer without distant metastasis were included in this retrospective evaluation. The following inclusion criteria were considered: (1) patients scheduled to receive CRT followed by TME surgery; (2) without metabolic diseases, such as diabetes mellitus or hyperthyroidism; (3) staged with 18F-FDG PET/CT before the nRCT and (4) informed consent was signed and obtained before the treatment. The neoadjuvant treatment consisted of intensity-modulated radiation therapy (IMRT) or 3- dimensional conformal RT (3DCRT) to a total dose of $50 \mathrm{~Gy}$ in 25 fractions or 50,4 Gy in 28 fractions delivered concurrently with 5-Fluorouracil (5-FU) based chemotherapy (5-FU plus leucovorin or oral capecitabine). Surgery was scheduled at a minimum of six weeks after the completion of nCRT. TME was mandatory, whereas the form of surgery-anterior resection or abdominal-perineal resection- and whether a temporary colostomy should be performed was decided by the surgeon. Postoperative maintenance 5FUbased chemotherapy was given according to the postoperative pathologic evaluations of patients. The study was approved by the institutional review board of Ege University Medical School \& Hospital.

Patients were pathologically staged according to the 2017 American Joint Committee on Cancer (AJCC), 8th edition.[15] Pathological tumor response was evaluated according to the 2010 AJCC tumor regression grade (TRG) system, which recorded the degree and the volume of the residual primary tumor cells. Details of AJCC TRG system are defined as follows: Grade 0, defined as no viable cancer cells; Grade 1, characterized by single or small groups of tumor cells; Grade 2, involves residual cancer outgrown by fibrosis, but fibrosis still predominates; and Grade 3, defined as the minimal or no tumor cells killed (Table 1). The nCRTsensitive patients were defined as those with TRG Grades $0-1$, while the resistant patients were defined as those with TRG Grades 2-3.

\section{Follow-up}

The patients were followed every three months for two years, then every six months up to five years and annually thereafter. Failure was defined as biopsy-proven recurrence or documented progression of disease in serial-imaging studies. Failure patterns were determined by follow-up imaging studies and were divided 
Table 1 Tumor regression grading system of the American Joint Committee on Cancer (15)

\begin{tabular}{lccc} 
Grade & Treatment response & Histologic features & Classification \\
\hline 3 & Poor & Minimal or no tumor cells killed, extensive residual tumor & Non-responder \\
2 & Minimal & Residual tumor overgrown by fibrosis & Non-responder \\
1 & Moderate & Single or small groups of cancer cells & Responder \\
0 & Complete & No viable cancer cells & Responder \\
\hline
\end{tabular}

into two groups: local and distant failure (including para-aortic and supraclavicular lymph nodes and also a distant organ).

\section{F-FDG PET/CT Technique}

The patients were imaged using a dedicated 18F-FDG PET/CT system, as previously described.[16] The patients fasted for at least six hours before intravenous administration of 370 to $555 \mathrm{MBq}$ (10-15 mCi) FDG. Preinjection blood glucose levels were measured to make sure that they were below $150 \mathrm{mg} / \mathrm{dL}$. During the distribution phase, the patients laid supine in a quiet room. Combined image acquisition began $60 \mathrm{~min}$ after FDG injection. The patients were scanned on a flatpanel, carbon-fiber composite table insert. First, an unenhanced CT scan (5-mm slice thickness) from the base of the skull to the inferior border of the pelvis was acquired using a standardized protocol $(140 \mathrm{kV}$ and 80 $\mathrm{mA}$ ). The subsequent PET scan was acquired in three-dimensional (3D) mode from the base of the skull to the inferior border of the pelvis ( 6 to 7 -bed positions, 3 min per position) without repositioning the patient on the table. CT and PET images were acquired with the patient breathing shallowly. Attenuation was corrected using the CT images. Areas of FDG uptake were categorized as malignant based on location, intensity, shape, size, and visual correlation with CT images to differentiate physiologic uptake from pathologic uptake. A lymph node was considered PET-positive if its FDG uptake was higher than blood pool activity or surrounding background tissues, depending on the size of the node.

\section{Image Analysis}

The tumor size is the maximum diameter measured on 18F-FDG PET/CT images. For each 18F-FDG PET/CT study, the SUVmax, SUVmean, MTV and TLG values of the primary tumor were measured. The SUV value higher than 2.5 was considered positive. A volumetric region of interest (ROI) around the outline of the primary tumor was placed on the axial 18F-FDG PET/CT images using the semi-automatic software. The ROI borders were manually adjusted by visual inspection of the primary tumor for avoiding an overlap on adjacent FDG-avid structures, and the 18F-FDG uptake of the urinary tract and bladder are excluded. MTV was defined as the regions equal to or

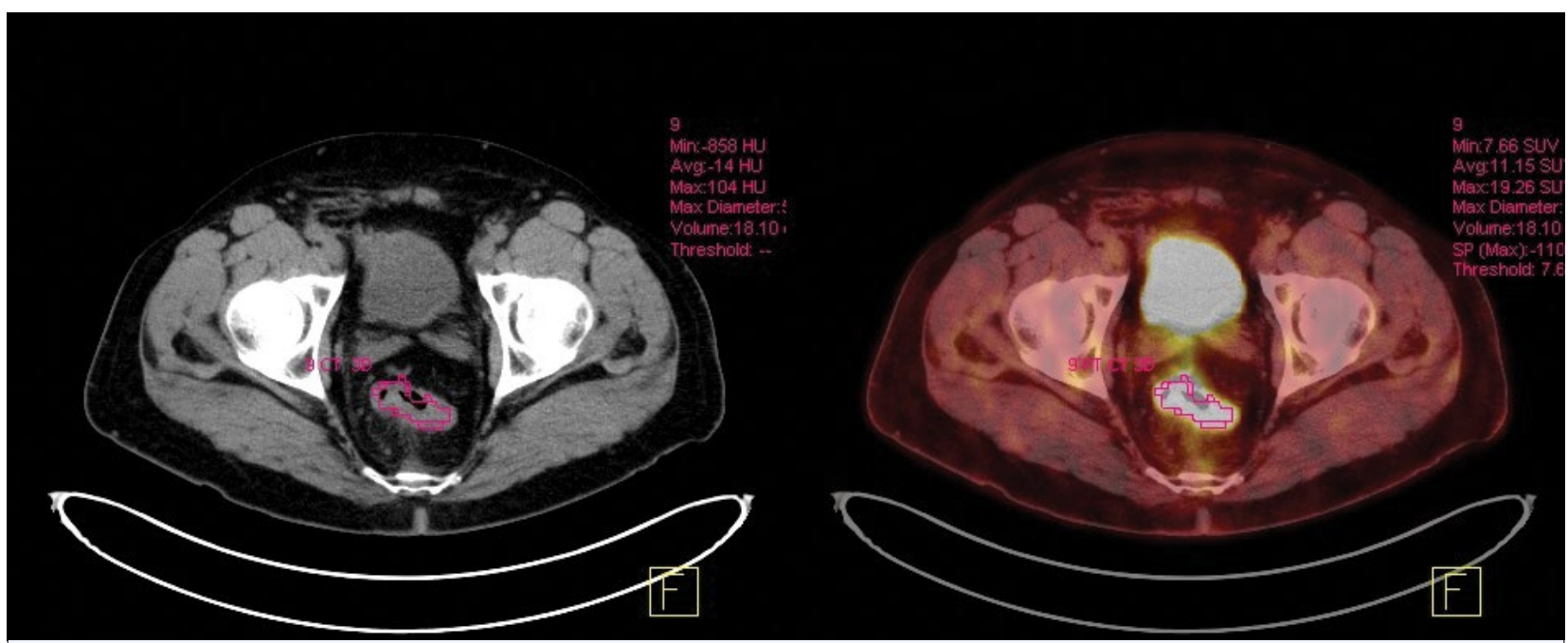

Fig. 1. Measurement of metabolic tumor volume (MTV) using a standardized uptake (SUV)-based automated contouring program. 
higher than $42 \%$ of the SUVmax (Fig. 1). To prevent the inclusion of adjacent normal structures, such as the bladder, lymph nodes, and the bowel, the tumor region was expanded from a single-seed voxel within the tumor via the region-growing morphologic operation. The PET parameters, including SUVmean, MTV, and the SUVmax, were automatically acquired with automatically generated ROI of the primary tumor. The TLG was calculated by multiplying SUVmean and MTV.

\section{Statistical Analysis}

All statistical analyses relied on standard software (SPSS v22; SPSS Inc. [IBM], Chicago, IL, USA). The time to event was calculated as the time interval from the date of diagnosis to the date of first finding on clinical or imaging examination that suggested disease recurrence. All time-related events (failure or death) were calculated from the first day of biopsy-proven diagnosis to the last follow-up or death. Disease-free survival (DFS) and overall survival (OS) rates were calculated using the Kaplan-Meier method. Correlations between parameters were calculated using the Pearson test. Variables shown to be significant or of borderline significance $(\mathrm{p}<0.1)$ were also selected for multivariate analysis. Multivariate analysis was performed using the Cox proportional hazards model, using covariates with a p-value less than 0.20 based on univariate analysis. The same results were observed after forward and backward inclusion in multivariate analysis. Receiver operating characteristic curves (ROC) were generated for the SUVmax, SUVmean, MTV and TLG values to determine the cut-off values for predicting recurrence and survival that yielded optimal sensitivity and specificity. Clinicopathological factors and follow-up data from our patient cohort were analyzed for correlations with SUVmax, SUVmean, MTV and TLG. All p-values $\leq 0.05$ were considered statistically significant.

\section{Results}

\section{Patient Characteristics}

Patient and tumor characteristics are presented in Table 2. All patients had adenocarcinoma histology and more than $80 \%$ had T3 and/or N1 disease. All patients were treated with concurrent chemotherapy, except 18 (17\%) patients. 5-FU+leucovorin or oral capecitabine was the chemotherapeutic agents used concomitantly. Among the patients receiving concurrent chemotherapy, 68 patients (64\%) received oral capecitabine, and
Table 2 Patient and tumor characteristics

\begin{tabular}{|c|c|}
\hline Characteristic & Number (\%) \\
\hline \multicolumn{2}{|l|}{ Age (year) } \\
\hline Median, range & $61(29-86)$ \\
\hline \multicolumn{2}{|l|}{ Sex } \\
\hline Female & $49(46 \%)$ \\
\hline Male & $57(54 \%)$ \\
\hline \multicolumn{2}{|l|}{ Location } \\
\hline Proximal+rectosigmoid & $18(17 \%)$ \\
\hline Middle rectum & $34(32 \%)$ \\
\hline Distal rectum & $54(51 \%)$ \\
\hline \multicolumn{2}{|l|}{ Histopathology } \\
\hline Adenocarcinoma & $102(96 \%)$ \\
\hline Signet ring cell carcinoma & $2(2 \%)$ \\
\hline Mucinous adenocarcinoma & $2(2 \%)$ \\
\hline \multicolumn{2}{|l|}{ Lymphovascular Invasion } \\
\hline$(+)$ & $20(18 \%)$ \\
\hline$(-)$ & $86(82 \%)$ \\
\hline \multicolumn{2}{|l|}{ Perineural invasion } \\
\hline$(+)$ & $20(18 \%)$ \\
\hline$(-)$ & $86(82 \%)$ \\
\hline \multicolumn{2}{|l|}{ CT Stage AJCC 2010} \\
\hline $\mathrm{T} 2$ & $6(6 \%)$ \\
\hline T3 & $90(85 \%)$ \\
\hline $\mathrm{T} 4$ & $10(9 \%)$ \\
\hline \multicolumn{2}{|l|}{ cN Stage AJCC 2010} \\
\hline No & $24(22 \%)$ \\
\hline N1 & 77 (73\%) \\
\hline N2 & $5(\% 5)$ \\
\hline \multicolumn{2}{|l|}{ Concomitant chemotherapy } \\
\hline Yes & $88(83 \%)$ \\
\hline No & $18(17 \%)$ \\
\hline \multicolumn{2}{|l|}{ Chemotherapy type } \\
\hline Capecitabine & $68(64 \%)$ \\
\hline 5-Fluorouracil+leucovorin & $20(19 \%)$ \\
\hline None & $18(17 \%)$ \\
\hline \multicolumn{2}{|l|}{ Radiotherapy technique } \\
\hline IMRT & $94(89 \%)$ \\
\hline 3DCRT & $12(11 \%)$ \\
\hline \multicolumn{2}{|l|}{ Pathological response to $\mathrm{nCRT}$} \\
\hline TRG 0/1 & $45(42.5 \%)$ \\
\hline TRG 2/3 & $61(57.5 \%)$ \\
\hline Complete response & $18(17 \%)$ \\
\hline \multicolumn{2}{|l|}{ FDG-PET/CT results, mean $\pm S D$} \\
\hline SUVmax & $16.9 \pm 9.6$ \\
\hline SUVmean & $9.6 \pm 6.3$ \\
\hline $\operatorname{MTV}\left(\mathrm{cm}^{3}\right)$ & $24.7 \pm 26.4$ \\
\hline TLG & $268.7 \pm 474.5$ \\
\hline
\end{tabular}

IMRT: Intensity modulated radiation therapy; 3DCRT: 3 dimentional radiation therapy; TRG: Tumor regression grade; TLG: Total lesion glycolysis; FDG-PET/CT: Fluorodeoxy glucose positron emission computed tomography; MTV: Metabolic tumor volume 
20 (19\%) received 5-FU+leucovorin. External beam RT was administered with 1.8-2 Gy daily fractions to a total dose of 50-50.4 Gy. IMRT technique was used in 94 patients $(89 \%)$, while the rest were treated with 3DCRT technique.

\section{Treatment Outcomes}

The median follow-up for all patients and surviving patients was 39.0 months (range, 6-103 months) and 40 months (range, 22-103 months), respectively. Of the 106 patients in our study cohort, $21(20 \%)$ patients developed local, locoregional, distant failure, or a combination of local/locoregional and distant failures. Of these, 16 (15\%) developed distant metastases, and five (5\%) patients had local/locoregional failure.

At the time of the last follow-up, 94 patients (89\%) were alive (11 [10\%] patients with disease), and 12 patients $(11 \%)$ were dead.

Pathologic complete response (pCR) was defined as the absence of viable cancer cells in the resected specimen (ypT0N0). pCR was achieved in $17 \%$ of all cases (18/106). According to TRG system, grade $0-1$ and grade 2-3 responders were 45 patients $(42.5 \%)$ and 61 patients $(57.5 \%)$, respectively.

\section{8-F FDG PET/CT Findings}

The mean \pm SD SUVmax, SUVmean, MTV and TLG values were 16.9 \pm 9.6 (range, 3.6-60.2), 9.6 \pm 6.3 (range, 2.4-49.2), $24.7 \pm 26.4 \mathrm{~cm}^{3}$ (range, $2.8-160.5 \mathrm{~cm}^{3}$ ) and 268.7 \pm 474.5 (range, 21.8-3.092.0) for the entire group, respectively. There was a weak correlation between the SUVmax of the primary rectal tumor and MTV (Pearson correlation coefficient $[\mathrm{r}]=0.238 ; \mathrm{p}<0.001$ ), whereas the correlation between SUVmax of the primary rectal tumor and TLG were moderate $(r=0.538$; $\mathrm{p}<0.001)$. A weak correlation between tumor size and SUVmax $(r=0.248 ; \mathrm{p}<0.001)$, and a moderate correlation between tumor size and MTV $(r=0.489 ; \mathrm{p}<0.001)$, and TLG $(r=0.512 ; \mathrm{p}<0.001)$ were observed.

The cut-off values for SUVmax, SUVmean, MTV, and TLG determined from the ROC curves were $13.5 \mathrm{~g} / \mathrm{ml}$ [area under the curve $(\mathrm{AUC})=0.357$, $95 \%$ confidence interval $(\mathrm{CI})=0.185-0.529], 8.44 \mathrm{~g} /$ $\mathrm{ml}$ (AUC=0.366, 95\% CI=0.186- 0.545), $14.65 \mathrm{~cm}^{3}$ (AUC $=0.549, \quad 95 \% \quad \mathrm{CI}=0.348-0.750)$, and 117.00 (AUC $=0.445,95 \% \mathrm{CI}=0.255-0.636$ ), respectively. Patients were divided into groups based on their values for each factor being below (low group) and at or above (high group) the cut-off value. The low and high SUVmax, SUVmean, MTV, and TLG groups included 44 (41\%) and $62(59 \%)$ patients, $50(47 \%)$ and $56(53 \%)$ patients, $51(48 \%)$ and $55(52 \%)$ patients, and 48 (45\%) and $58(55 \%)$ patients, respectively.

We could not find any significant correlation between FDG-PET/CT parameters and treatment response to nCRT according to TRG system.

\section{Correlations between FDG-PET/CT Parameters and Patient/Tumor Characteristics}

Neither SUVmax nor SUVmean was affected by patient and tumor characteristics. On the other hand, MTV and TLG were significantly higher in patients with larger tumors $(>3 \mathrm{~cm})(\mathrm{p}=0.031$, and $\mathrm{p}=0.002$ respectively), advanced $\mathrm{cT}$ stage $(\mathrm{p}=0.004$, and $\mathrm{p}=0.043$ respectively), and other than distal locations ( $\mathrm{p}=0.049$, and $\mathrm{p}=0.037$ respectively) (Table 3 ).

\section{Survival Analysis and Prognostic Factors}

The 3- and 5-year overall survival (OS) rates were $92 \%$ and $80 \%$, respectively (Fig. 2a). On univariate analysis, perineural invasion (PNI), concomitant CT usage, posttreatment $\mathrm{T}$ (ypT) stage, ypN stage, and any response to treatment according to TRG system were prognostic factors for OS.

The 3- and 5-year local recurrence-free survival (LRFS) rates were 95\% and 92\%, respectively (Fig. 2b). On univariate analysis, the complete nodal response was the only prognostic factor for LRFS.

The 3- and 5-year distant metastasis-free survival (DMFS) rates were $87 \%$ and $38 \%$, respectively (Fig. 2c). On univariate analysis, lymphovascular invasion (LVI), posttreatment stage, ypN stage and treatment response according to the TRG system were prognostic factors for DMFS.

On multivariate analysis, we could not find any statistically significant prognostic factor both for LRFS and DMFS. On the other hand, posttreatment extensive stage of disease $(\mathrm{p}=0.013)$, absence of concomitant CT $(\mathrm{p}=0.012)$, MTV $\geq 14.65 \mathrm{~cm}^{3}(\mathrm{p}=0.008)$, and TLG $\geq 117.00$ ( $\mathrm{p}=0.023$ ) were unfavorable prognostic factors for OS (Table 4).

\section{Discussion}

Stage of disease, tumor size, and concomitant CT application are the strongest prognostic factors in patients with locally advanced rectal cancer.[4,17] However, FDG-PET/CT parameters were also considered as prognostic factors in some series for better assessing the tumor characteristics in patients treated both with the neoadjuvant and adjuvant settings. Thus, FDG-PET/ $\mathrm{CT}$ is a valuable tool that incorporates metabolic tumor 
Prognostic PET/CT Parameters for Rectal Cancer

Table 3 Correlations between metabolic parameters of FDG-PET/CT and patient/tumor characteristics

\begin{tabular}{|c|c|c|c|c|c|c|c|c|c|c|}
\hline Variables & $\mathbf{n}$ & $\%$ & $\begin{array}{c}\text { SUVmax } \\
\text { (Mean士SD) }\end{array}$ & $\mathbf{p}$ & $\begin{array}{l}\text { SUVmean } \\
\text { (Mean士SD) }\end{array}$ & $\mathbf{p}$ & $\begin{array}{c}\text { MTV } \\
(\text { Mean士SD) }\end{array}$ & $\mathbf{p}$ & $\begin{array}{c}\text { TLG } \\
\text { (Mean士SD) }\end{array}$ & $\mathbf{p}$ \\
\hline \multicolumn{11}{|l|}{ Age (years) } \\
\hline$\leq 65$ & 71 & 67 & $17.4 \pm 10.1$ & 0.493 & $9.9 \pm 6.9$ & 0.460 & $24.8 \pm 25.4$ & 0.974 & $274.5 \pm 467.5$ & 0.858 \\
\hline$>65$ & 35 & 33 & $16.0 \pm 8.7$ & & $9.0 \pm 4.9$ & & $24.5 \pm 28.5$ & & $256.9 \pm 495.0$ & \\
\hline \multicolumn{11}{|l|}{ Sex } \\
\hline Female & 49 & 46 & $17.8 \pm 9.6$ & 0.416 & $10.4 \pm 7.1$ & 0.227 & $23.3 \pm 27.0$ & 0.617 & $237.5 \pm 344.0$ & 0.533 \\
\hline Male & 57 & 54 & $16.2 \pm 9.7$ & & $8.9 \pm 5.4$ & & $25.9 \pm 26.0$ & & $295.5 \pm 564.9$ & \\
\hline \multicolumn{11}{|c|}{ Tumor size $(\mathrm{cm})$} \\
\hline$\leq 3$ & 32 & 30 & $16.7 \pm 10.1$ & 0.231 & $9.8 \pm 6.9$ & 0.315 & $12.7 \pm 10.3$ & 0.031 & $120.8 \pm 480.4$ & 0.002 \\
\hline$>3$ & 74 & 70 & $18.1 \pm 9.7$ & & $10.9 \pm 7.8$ & & $26.8 \pm 25.8$ & & $284.8 \pm 497.5$ & \\
\hline \multicolumn{11}{|c|}{ Tumor location } \\
\hline Distal & 54 & 51 & $15.6 \pm 8.2$ & 0.152 & $8.8 \pm 4.5$ & 0.145 & $19.7 \pm 21.8$ & 0.049 & $189.1 \pm 384.5$ & 0.037 \\
\hline Other & 52 & 49 & $18.3 \pm 10.9$ & & $10.5 \pm 7.6$ & & $29.8 \pm 29.7$ & & $351.3 \pm 544.2$ & \\
\hline \multicolumn{11}{|l|}{ cT Stage } \\
\hline $\mathrm{T} 2 / 3$ & 96 & 91 & $17.2 \pm 9.9$ & 0.514 & $9.8 \pm 6.4$ & 0.577 & $22.5 \pm 22.5$ & 0.004 & $244.5 \pm 448.0$ & 0.043 \\
\hline $\mathrm{T} 4$ & 10 & 9 & $15.0 \pm 7.6$ & & $8.6 \pm 4.6$ & & $47.2 \pm 46.5$ & & $519.1 \pm 671.0$ & \\
\hline \multicolumn{11}{|l|}{ cN Stage } \\
\hline No & 24 & 23 & $14.6 \pm 6.9$ & 0.181 & $8.3 \pm 4.0$ & 0.244 & $30.4 \pm 34.6$ & 0.229 & $290.3 \pm 462.0$ & 0.801 \\
\hline $\mathrm{N} 1 / 2$ & 72 & 77 & $17.6 \pm 10.2$ & & $10.0 \pm 6.7$ & & $23.0 \pm 23.4$ & & $262.4 \pm 480.7$ & \\
\hline \multicolumn{11}{|c|}{ Treatment response } \\
\hline TRG 0/1 & 45 & 43 & $16.7 \pm 8.8$ & 0.802 & $9.4 \pm 5.2$ & 0.801 & $20.6 \pm 17.7$ & 0.178 & $221.7 \pm 312.3$ & 0.384 \\
\hline TRG 2/3 & 61 & 57 & $17.1 \pm 10.3$ & & $9.8 \pm 7.0$ & & $27.6 \pm 31.1$ & & $303.3 \pm 565.3$ & \\
\hline
\end{tabular}

FDG-PET/CT: Fluorodeoxy glucose positron emission computed tomography; 3DCRT: 3 dimentional radiation therapy; TRG: Tumor regression grade; TLG: Total lesion glycolysis

Table 4 Multivariate analysis of prognostic factors for overall survival

\begin{tabular}{lccc} 
Variables & Risk factors & HR (95\% Cl) & p \\
\hline Posttreatment stage of disease & Extensive vs early disease & $1.99(1.18-3.20)$ & 0.013 \\
Concomitant CT & Absent vs present & $2.12(1.15-3.45)$ & 0.012 \\
MTV & $\geq 14.65$ vs $<14.65$ & $2.10(1.24-3.54)$ & 0.008 \\
TLG & $\geq 117$ vs $<117$ & $1.84(1.20-2.89)$ & 0.023
\end{tabular}

HR: Hazad ratio; CT: Chemoterapy; MTV: Metabolic tumor volume; TLG: Total lesion glycolysis

function with anatomical localization, and also can be accepted as an important imaging modality showing both distant metastasis and regional extension of disease, providing an opportunity for accurate treatment of these patients. The present study investigated the prognostic significance of FDG-PET/CT parameters (SUVmax, SUVmean, MTV, and TLG) in locally advanced non-metastatic rectal cancer patients treated with nCRT.

Several studies investigated the predictive role of FDG-PET/CT for metabolic tumor response in LARC after nCRT.[18-24] Sun et al.[22] concluded that volumetric FDG-PET/CT parameters could be accepted as important tools for evaluating the tumor response to nCRT in LARC patients. They compared pre- and post- treatment FDG-PET/CT images, and they showed that the percentage change in MTV and TLG between pre- and posttreatment FDG-PET/CT scans could be used for giving more reliable prediction of the pathological response.[22] On the other hand, Hatt et al.[23] reported that early prediction of tumor response to nCRT in LARC with FDG-PET/CT could be misleading because of the limited reproducibility of FDG-PET scans.[23] We could not have a chance to compare pre- and post- treatment FDG-PET/CT due to the retrospective nature of our trial. We compared the pretreatment FDG-PET/CT findings with tumor response to nCRT, but we could not find any relation between them. 



Fig. 2. Overall survival (a), local recurrence-free survival (b), and distant metastasis-free survival (c) curves for the entire cohort.

The prognostic role of FDG-PET/CT parameters is the other highly evaluated issue for many cancer types. Huang et al.[25] investigated the prognostic value of repeated FDG PET/CT for early prediction of survival in locally advanced non-small cell lung cancer (NSCLC) patients treated with concomitant CRT. They showed that the MTV of the primary tumor has the potential to become a valuable prognostic biomarker for survival outcome in NSCLC patients, and a decrease in MTV



Fig. 2. Overall survival (a), local recurrence-free survival (b), and distant metastasis-free survival (c) curves for the entire cohort.

by the primary tumor correlates with a higher longterm OS.[25]

Bang et al.[16] evaluated metabolic and textural parameters from pretreatment FDG-PET/CT scans for the prediction of treatment response and 3-year DFS in patients with LARC. They performed a retrospective analysis of FDG-PET/CT scans of 74 patients, and they used the TRG system for treatment response evaluation similar to the present study. They concluded that metabolic and textural parameters could be used to evaluate tumor heterogeneity for the prediction of nCRT response and recurrence in LARC.[16] However, we could not find any significant correlation between FDG-PET/CT parameters and treatment response to nCRT according to the TRG system.

Most recently, Okuno et al.[26] investigated the FDG-PET/CT parameters concerning their contribution to the prediction of pathological complete response or prognosis after nCRT. Ninety-one consecutive patients with LARC were included in their study. They performed both pre- and posttreatment FDG-PET/CT scans. They found that high TLG after nCRT was strongly associated with a worse prognosis for the patients with LARC, and concluded that TLG after treatment might be a promising preoperative predictor of recurrence and death.[26] The current study showed that MTV $\geq 14.65 \mathrm{~cm}^{3}$ and TLG $\geq 117.00$ on pretreatment FDG-PET/CT were related to worse 
OS in LARC patients treated with nCRT. We could not show any significant relation with the well-known prognostic factors, such as SUVmax and SUVmean. However, MTV and TLG have been developed to calculate the metabolic activity in the whole tumor. MTV is a well-known prognostic factor in various cancers because it represents the dual characteristics of tumor volume and the degree of FDG uptake by the tumor.[27] TLG has been proposed as a more accurate parameter because it considers both SUVmean and MTV.[28] There exists a controversial debate regarding which parameter -MTV or TLG- is superior for predicting prognosis. In the current study, we found that both MTV and TLG were associated with OS in LARC patients, and this finding might be explained that volumetric FDG-PET/CT parameters reflect the metabolic burden of cancer.[29,30] We should consider additional systemic treatments for patients with high metabolic tumor burden.

There are some limitations concerning the usage of FDG-PET/CT as a prediction and/or prognostic tool for patients with LARC. We should interpret the FDG-PET/CT parameters with caution because different cut-off value determination methods were used in the literature and different SUV threshold values for defining MTV and TLG were preferred for those limited number of studies. The optimal cut-off values for SUVmax, SUVmean, MTV and TLG have not yet been established. In our study, we used ROC curve analysis to determine the thresholds as previous studies have been conducted. However, they could not give any exact cut-off value for FDG-PET/CT derived parameters. Despite all uncertainties, it can be concluded that higher MTV and TLG may be a sign of more aggressive behavior for LARC and we should consider more intensive treatment approaches and follow-up schedules for these groups of patients.

Our study has some limitations. The retrospective nature of the study and lack of PET/CT fter nCRT are the main limitations. In addition, we measured the tumor size in PET-CT images. Measuring the tumor size in T2-weighted MRI images should be more reliable. However, our findings, which were based on a larger and more homogenous patient population treated with nCRT in modern RT era, and our study cohort, which was considerably larger than those of previous reports, would be more helpful for evaluating the significance of FDG PET/CT metabolic parameters for survival and assessing the correlations of these parameters with other risk factors.

\section{Conclusion}

Historically proven prognostic factors, such as stage and concomitant CT applications, are still the most reliable indicators related to the treatment outcomes for the patients with LARC. In our study, we demonstrated that higher MTV and TLG, which reflect tumor burden, is an important issue in terms of treatment outcomes. However, the clinical benefits of using FDG PET/CT metabolic parameters to predict high-risk patients and eventually to change treatment strategy in LARC patients still need further clarification.

Peer-review: Externally peer-reviewed.

Conflict of Interest: All authors have declared that they have no conflict of interest.

Ethics Committee Approval: This study approval is obtained from local ethical committee.

Financial Support: There is no financial support.

Authorship contributions: Concept - F.S., R.S., A.O.; Design - F.S., A.O., R.S.; Supervision - F.S., D.Y., S.O.; Funding

- None; Materials - None; Data collection and/or processing - F.S., A.O., R.S.; Data analysis and/or interpretation - F.S., A.O., R.S.; Literature search - F.S., D.Y., S.O.; Writing - F.S., D.Y.; Critical review - F.S., S.O.

\section{References}

1. van de Velde CJ, Aristei C, Boelens PG, Beets-Tan RG, Blomqvist L, Borras JM, et al. EURECCA colorectal: multidisciplinary mission statement on better care for patients with colon and rectal cancer in Europe. Eur J Cancer 2013;49(13):2784-90.

2. Valentini V, Aristei C, Glimelius B, Minsky BD, Beets-Tan R, Borras JM, et al. Multidisciplinary rectal cancer management: 2nd European rectal cancer consensus conference (EURECA-CC2). Radiother Oncol 2009;92(2):148-63.

3. Rödel C, Liersch T, Becker H, Fietkau R, Hohenberger W, Hothorn T, et al. Preoperative chemoradiotherapy and postoperative chemotherapy with fluorouracil and oxaliplatin versus fluorouracil alone in locally advanced rectal cancer: initial results of the German CAO/ARO/AIO-04 randomised phase 3 trial. Lancet Oncol 2012;13(7):679-87.

4. Sauer R, Liersch T, Merkel S, Fietkau R, Hohenberger W, Hess C, et al. Preoperative versus postoperative chemoradiotherapy for locally advanced rectal cancer: results of the German CAO/ARO/AIO-94 randomized phase III trial after a median follow-up of 11 years. J Clin Oncol 2012;30(16):1926-33. 
5. Onaitis MW, Noone RB, Hartwig M, Hurwitz H, Morse $M$, Jowell $P$, et al. Neoadjuvant chemoradiation for rectal cancer: analysis of clinical outcomes from a 13-year institutional experience. Ann Surg 2001;233(6):77885.

6. Kaminsky-Forrett MC, Conroy T, Luporsi E, Peiffert D, Lapeyre M, Boissel P, et al. Prognostic implications of downstaging following preoperative radiation therapy for operable T3-T4 rectal cancer. Int J Radiat Oncol Biol Phys 1998;42(5):935-41.

7. Kuremsky JG, Tepper JE, McLeod HL. Biomarkers for response to neoadjuvant chemoradiation for rectal cancer. Int J Radiat Oncol Biol Phys 2009;74(3):673-88.

8. Brown G, Davies S, Williams GT, Bourne MW, Newcombe RG, Radcliffe AG, et al. Effectiveness of preoperative staging in rectal cancer: digital rectal examination, endoluminal ultrasound or magnetic resonance imaging? Br J Cancer 2004;91(1):23-9.

9. Joye I, Deroose CM, Vandecaveye V, Haustermans K. The role of diffusion-weighted MRI and (18)F-FDG $\mathrm{PET} / \mathrm{CT}$ in the prediction of pathologic complete response after radiochemotherapy for rectal cancer: a systematic review. Radiother Oncol 2014;113(2):158-65.

10. Kim SH, Song BI, Kim BW, Kim HW, Won KS, Bae SU, et al. Predictive Value of [18F]FDG PET/CT for Lymph Node Metastasis in Rectal Cancer. Sci Rep 2019;9(1):4979.

11.van Stiphout RG, Valentini V, Buijsen J, Lammering G, Meldolesi E, van Soest J, et al. Nomogram predicting response after chemoradiotherapy in rectal cancer using sequential PETCT imaging: a multicentric prospective study with external validation. Radiother Oncol 2014;113(2):215-22.

12. Hatt M, Visvikis D, Albarghach NM, Tixier F, Pradier O, Cheze-le Rest C. Prognostic value of 18F-FDG PET image-based parameters in oesophageal cancer and impact of tumour delineation methodology. Eur J Nucl Med Mol Imaging 2011;38(7):1191-202.

13. Hyun SH, Choi JY, Shim YM, Kim K, Lee SJ, Cho YS, et al. Prognostic value of metabolic tumor volume measured by $18 \mathrm{~F}$-fluorodeoxyglucose positron emission tomography in patients with esophageal carcinoma. Ann Surg Oncol 2010;17(1):115-22.

14. Zhang C, Chen Y, Xue H, Zheng P, Tong J, Liu J, et al. Diagnostic value of FDG-PET in recurrent colorectal carcinoma: a meta-analysis. Int J Cancer 2009;124(1):167-73.

15. National Comprehensive Cancer Network. Available at: https://www.nccn.org/professionals/physician_gls/ pdf/rectal.pdf assessed at 06/05/2019. Accessed Feb 4, 2020.

16. Bang JI, Ha S, Kang SB, Lee KW, Lee HS, Kim JS, et al. Prediction of neoadjuvant radiation chemotherapy response and survival using pretreatment [(18)F]FDG
$\mathrm{PET} / \mathrm{CT}$ scans in locally advanced rectal cancer. Eur J Nucl Med Mol Imaging 2016;43(3):422-31.

17. Kim SJ, Chang S. Volumetric parameters changes of sequential 18F-FDG PET/CT for early prediction of recurrence and death in patients with locally advanced rectal cancer treated with preoperative chemoradiotherapy. Clin Nucl Med 2015;40(12):930-5.

18. Everaert H, Hoorens A, Vanhove C, Sermeus A, Ceulemans G, Engels B, et al. Prediction of response to neoadjuvant radiotherapy in patients with locally advanced rectal cancer by means of sequential 18FDGPET. Int J Radiat Oncol Biol Phys 2011;80(1):91-6.

19. Herrmann K, Bundschuh RA, Rosenberg R, Schmidt S, Praus C, Souvatzoglou M, et al. Comparison of different SUVbased methods for response prediction to neoadjuvant radiochemotherapy in locally advanced rectal cancer by FDG-PET and MRI. Mol Imaging Biol 2011;13(5):1011-9.

20. Hur H, Kim NK, Yun M, Min BS, Lee KY, Keum KC, et al. 18Fluoro-deoxy-glucose positron emission tomography in assessing tumor response to preoperative chemoradiation therapy for locally advanced rectal cancer. J Surg Oncol 2011;103(1):17-24.

21. Kim JW, Kim HC, Park JW, Park SC, Sohn DK, Choi HS, et al. Predictive value of (18)FDG PET-CT for tumour response in patients with locally advanced rectal cancer treated by preoperative chemoradiotherapy. Int J Colorectal Dis 2013;28(9):1217-24.

22. Sun W, Xu J, Hu W, Zhang Z, Shen W. The role of sequential $18(\mathrm{~F})$-FDG PET/CT in predicting tumour response after preoperative chemoradiation for rectal cancer. Colorectal Dis 2013;15(5):e231-8.

23. Hatt M, van Stiphout R, le Pogam A, et al. Early prediction of pathological response in locally advanced rectal cancer based on sequential 18F-FDG PET. Acta Oncol. 2013;52:619-626.

24. Altini C, Niccoli Asabella A, De Luca R, Fanelli M, Caliandro C, Quartuccio N, et al. Comparison of 18FFDG PET/CT methods of analysis for predicting response to neoadjuvant chemoradiation therapy in patients with locally advanced low rectal cancer. Abdom Imaging 2015;40(5):1190-202.

25. Huang W, Fan M, Liu B, Fu Z, Zhou T, Zhang Z, et al. Value of metabolic tumor volume on repeated 18F-FDG PET/CT for early prediction of survival in locally advanced non-small cell lung cancer treated with concurrent chemoradiotherapy. J Nucl Med 2014;55(10):1584-90.

26. Okuno T, Kawai K, Koyama K, Takahashi M, Ishihara S, Momose T, et al. Value of FDG-PET/CT Volumetry After Chemoradiotherapy in Rectal Cancer. Dis Colon Rectum 2018;61(3):320-7.

27. Chung MK, Jeong HS, Park SG, Jang JY, Son YI, Choi JY, et al. Metabolic tumor volume of [18F]-flu- 
orodeoxyglucose positron emission tomography/ computed tomography predicts short-term outcome to radiotherapy with or without chemotherapy in pharyngeal cancer. Clin Cancer Res 2009;15(18):5861-8.

28. Larson SM, Erdi Y, Akhurst T, Mazumdar M, Macapinlac HA, Finn RD, et al. Tumor treatment response based on visual and quantitative changes in global tumor glycolysis using PET-FDG imaging. The visual response score and the change in total lesion glycolysis. Clin Positron Imaging 1999;2(3):159-71.
29. Jo HJ, Kim SJ, Lee HY, Kim IJ. Prediction of survival and cancer recurrence using metabolic volumetric parameters measured by $18 \mathrm{~F}-\mathrm{FDG} \mathrm{PET} / \mathrm{CT}$ in patients with surgically resected rectal cancer. Clin Nucl Med 2014;39(6):493-7.

30. Ogawa S, Itabashi M, Kondo C, Momose M, Sakai S, Kameoka S. Prognostic Value of Total Lesion Glycolysis Measured by 18F-FDG-PET/CT in Patients with Colorectal Cancer. Anticancer Res 2015;35(6):3495500 . 